\title{
The Influence of Dopamine on Spatial Vision
}

\author{
J. P. HARRIS*, J. E. CALVERT* , J. A. LEENDERTZ**, and O. T. PHILLIPSON** \\ Bristol
}

\begin{abstract}
Summary
Contrast thresholds for, and contrast matches between, stationary gratings of three spatial frequencies $(0.5,2$, and $8 \mathrm{c} / \mathrm{deg})$ were measured on eight subjects with a history of schizophrenia, just before, and again two to three days after, a therapeutic injection of depot neuroleptic. The drug enhanced sensitivity at the low, and reduced it at the medium and high spatial frequency. After injection, subjects required more contrast to match the apparent contrast of the high, and less contrast to match that of the low, to that of the medium spatial frequency. Pupillary measurements suggested that these effects were not due to drug-induced changes in pupil size. The results are discussed in terms of the functional role of dopamine in the retina, and a possible application in therapy for amblyopia.
\end{abstract}

\section{Potential roles for drugs in therapy for amblyopia}

Current treatment for amblyopia is environmental: the 'good' eye is covered for a variable period each day, so that the patient is 'forced to use' the amblyopic eye. Such treatment is not always effective, and the patient may be left with a permanent visual deficit in the affected eye. A possible approach to improvement, which may be worth considering, is the use of drugs. Animal studies suggest two ways in which pharmaceutical agents may be useful in amblyopic therapy: first, it may be possible to elongate or re-create the human visual 'critical period', since there are reports that one can 're-plasticise' cat visual cortex; and secondly, since certain neuromodulators appear to be involved in the receptive field properties of visual neurones, it may be possible to enhance the effects of environmental therapy by altering their activity.

At present such ideas are 'science fiction': no-one knows whether it is possible to plasticise human neural systems (and what the unwanted consequences would be), and the study of the role of neuromodulators in human vision is in its infancy. The second approach may have some promise, however, but it presupposes an understanding of visual neurotransmitter function, and the present study is an attempt to extrapolate from the results of anatomical and pharmacological work in animals to visual perception in man.

\section{Dopamine in vision}

Dopamine (DA) is an important neurotransmitter in the CNS as a whole, and is found at several sites where it may influence visual function directly. For example, it exists in the retina in many species, including $\operatorname{man}^{1}$, and there is a dopaminergic projection from the midbrain to visual cortex in the cat. ${ }^{2}$ The function of the cortical projection is unknown, but the role of retinal dopamine has been widely

From: *Perceptual Systems Research Centre, Department of Psychology, Bristol; and ${ }^{* *}$ Department of Anatomy, School of Medical Sciences, University of Bristol, Bristol.

Correspondence to: J. P. Harris, Department of Psychology, University of Reading, Early Gate, Whiteknights, Reading RG26 2AL. 
studied. DA amacrines secrete dopamine in a graded manner as retinal illuminance is raised $^{3,4,5}$; DA produces a change in the centre/surround organisation of some receptive fields ${ }^{6}$; and it can produce a shift of gain in the response of ganglion cells. ${ }^{7}$ From evidence like this it has been suggested that DA is involved in the re-organisation of receptive field properties which accompanies changes of retinal illuminance. ${ }^{8}$

In man, sensitivity to medium and high spatial frequencies is progressively lost as retinal illuminace is lowered, and the contrast sensitivity function changes from band-pass, with a peak at medium spatial frequencies, to low pass. ${ }^{9}$ Physiologically, these changes may correspond to a reduction in contrast gain in retinal ganglion cells. If the reduction were more rapid for small receptive fields, or if they were active only at and above medium luminances, the psychophysical data would be explained. ${ }^{10}$ On the assumption that, in man, this process involves DA amacrines, one might expect that interference with DA function would change the perception of contrast.

\section{Evidence for a role for dopamine in contrast perception}

Since dopamine is secreted by amacrines as luminance rises, a working hypothesis is that a reduction in DA activity in man would produce an effect on contrast perception like that of lowering luminance, and an increase in DA activity would have the opposite effect. Some evidence for this view already exists: in Parkinson's disease (PD), losses of contrast sensitivity for medium and high spatial frequencies, and an enhancement of sensitivity at low spatial frequencies, have been reported. ${ }^{11}$ Such changes need not involve the retina, but studies of the latencies of the $\mathrm{VEP}^{12}$ and $\mathrm{ERG}^{13}$ suggest a retinal DA abnormality in the illness. Conversely, Domenici et al. ${ }^{14}$ found that dopaminergic drugs can enhance contrast sensitivity for medium spatial frequencies in normal individuals. At contrast threshold, the interference with DA behaves as predicted from the luminance adaptation hypothesis. Does DA have similar effects above threshold? One way to investigate this is to use a visual test which is known to depend upon contrast level in normal vision. The size of the tilt aftereffect (TAE - the apparent change of orientation of a line or grating which can follow prior inspection of a line or grating of a slightly different orientation) is known to depend on stimulus contrast. ${ }^{15}$ When the adapting and test gratings $(2 \mathrm{c} / \mathrm{deg})$ are of low contrast $(10 \%)$ and the test grating is of short duration $(100 \mathrm{~ms})$, the effects of $200 \mathrm{mg}$ oral L-dopa are almost identical to raising the contrast to $30 \%{ }^{16}$ The opposite effect is found when PD patients are compared with age-matched controls. ${ }^{17}$ These two experiments suggest that DA may indeed be involved in the supra-threshold contrast gain of medium spatial frequency channels.

The present study is an attempt to demonstrate, above threshold, the kind of shift in the contrast response function found at threshold in $\mathrm{PD},{ }^{11}$ namely a loss at medium and high spatial frequencies and an enhancement at low spatial frequencies. The experimental technique used is contrast matching, in which a 'standard' grating of some contrast is presented, and the subject's task is to match its apparent contrast by adjusting the contrast of a 'variable' grating of a different spatial frequency. In the experiment to be reported, the spatial frequency of the standard grating was always $2 \mathrm{c} / \mathrm{deg}$, while that of the variable was either 2,8 or $0.5 \mathrm{c} / \mathrm{deg}$. If the effect reported by Bodis-Wollner also holds above threshold, then, when DA is depleted, less contrast should be needed to match the apparent contrast of the low $(0.5 \mathrm{c} / \mathrm{deg})$ grating to that of the medium $(2 \mathrm{c} / \mathrm{deg})$, and more to match the contrast of the high $-(8 / \mathrm{deg})$ grating to the medium. The $2 \mathrm{c} / \mathrm{deg}$ variable was used to check on how well subjects could make contrast matches: if the matches were poor even when the two gratings had the same spatial frequency, it would reduce confidence in the matches made when they were not. Contrast thresholds and pupil diameter were also measured: the pupil size is one determinant of retinal illuminance which is known to affect the perception of contrast.

\section{Methods}

Apparatus

A purpose built digital generator ${ }^{18}$ produced the necessary waveforms to generate stationary gratings with a sinusoidal luminance pro- 
file on the face of a Tektronix 608 display oscilloscope. The frame rate was $100 \mathrm{~Hz}$, and the generator was programmed between frames by an Apple II microcomputer. The oscilloscope screen was divided electronically into two around its vertical midline, so that vertical gratings whose spatial frequency and contrast were independently variable (with 10 bit resolution) could be presented on both sides of the screen. The dimensions of the whole screen were six degrees wide by five degrees high at the viewing distance of 114 $\mathrm{cm}$, which was maintained with a chin rest. The mean luminance was always $120 \mathrm{~cd} / \mathrm{m}^{2}$ The vertical midline of the screen was covered with a strip of black card 0.5 degrees wide, which was expanded in the centre to form a disc two degrees in diameter. A small hole in the centre of the disc served as fixation point. The object of the disc and fixation point was to prevent the subject using his fovea in the task, since in rhesus monkey DA amacrines parallel the rods in density, being sparse or absent in the fovea and peaking some degrees into the periphery. ${ }^{19}$ A similar organisation appears to exist in man, and to be disrupted in Parkinson's disease. ${ }^{20}$ The screen was surrounded by a dark surround, and the experimental room was dimly lit.

\section{Procedure}

\section{Contrast thresholds}

The subject was instructed to fixate the central point throughout the procedure. On each presentation, at an auditory signal (tone) from the computer, the subject adjusted the contrast of the initially blank screen until the grating, which appeared on one side only, could just be detected. Contrast was adjusted with push-buttons, one of which increased, while the other decreased, contrast. Simultaneous depression of both buttons informed the computer, which acknowledged with a tone, that the subject was satisfied with the threshold setting. After an interval of five seconds, the next presentation began. The side of the screen on which the grating was presented, and its spatial frequency $(0.5,2$ or $8 \mathrm{c} / \mathrm{deg}$ ), were selected at random on each presentation. Five threshold settings were made at each spatial frequency for each side of the screen.

\section{Contrast matching}

As for contrast thresholds, the subject adjusted the contrast of a grating on one side of the screen. However, a standard $2 \mathrm{c} / \mathrm{deg}$ grating was presented on the other side of the screen, and the subject had to match its apparent contrast by adjusting the variable, whose spatial frequency was either $0.5,2$ or $8 \mathrm{c} / \mathrm{deg}$. The contrast of the standard was either 10, 20, 30 or $40 \%$. Five presentations of the standard grating at each contrast were made on each side of the screen, paired with each of the three variable spatial frequencies. The side of the standard, and its contrast, were chosen at random on each presentation, and was signalled to the subject by turning on one of two red LEDs, each positioned above one side of the screen.

\section{Pupillometry}

The subject adapted to a ring of LEDs at a luminance of about $0.5 \mathrm{~cd} / \mathrm{m}^{2}$ in an otherwise dark room for 10 minutes. The LEDs were then brightened in square-wave fashion to about $390 \mathrm{~cd} / \mathrm{m}^{2}$ for five seconds to produce the light reflex. These pulses were presented every 20 seconds for five minutes, and the luminance fell to $0.5 \mathrm{~cd} / \mathrm{m}^{2}$ between flashes. Successive pupillograms were then averaged, and the maximum and minimum pupil diameter found by measuring electronically in the video signal produced by an infra-red TV camera the time (and so the distance) between the voltage steps corresponding to the edges of the pupil. The apparatus and procedure are more fully described by Beaumont et al. ${ }^{21}$

\section{Subjects}

The eight subjects (three female, mean age 44.4 years) all had a history which included a diagnosis of schizophrenia. They were stabilised on depot injections of neuroleptic (Stelazine, Modecate or Haldol) with a mean inter-injection interval of three weeks. They were all working in an industrial therapy workshop, and living in the community or in sheltered hostels. The validity of the diagnosis of schizophrenia is irrelevant to the design of the study, which was concerned with the possible visual effects of the neuroleptic injection.

The data to be presented were gathered on 
two testing occasions, one on the day before an injection and one two to three days after an injection. For half the subjects, the first testing session was pre-, and for half, post-injection. So that they could gain experience of the tests and the laboratory environment, five subjects were tested on two, and three subjects on one, prior occasions. The data from these sessions were excluded from the analysis.

Blood levels (and so presumably CNS levels) of neuroleptic vary between injections, ${ }^{22}$ reaching a maximum two to three days after an injection and declining exponentially until just after the next injection. The times of testing were chosen to correlate with these differences.

\section{Results}

\section{Contrast Matching}

The matches found, when the task was to match the contrast of one $2 \mathrm{c} / \mathrm{deg}$ grating to that of another $2 \mathrm{c} / \mathrm{deg}$ grating, are shown in Figure 1. If performances were perfect, the matched contrasts would fall on a straight line

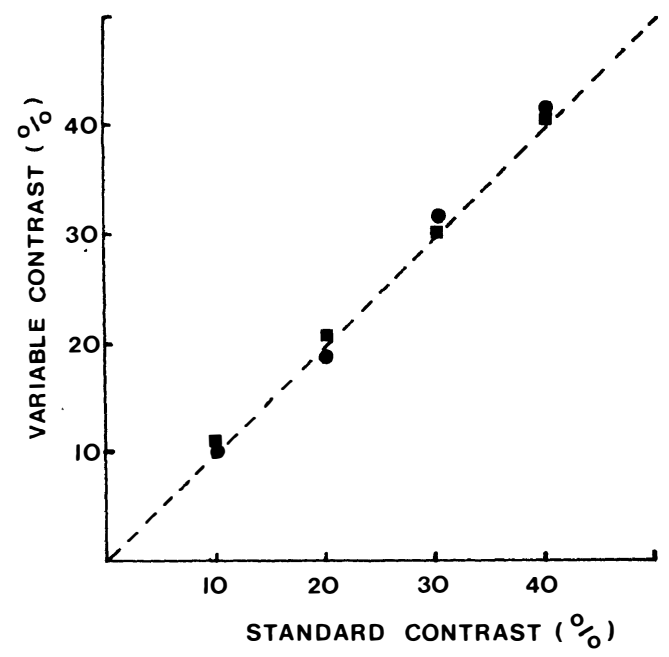

Fig. 1. The contrasts required to match the apparent contrast of a 'variable' $2 \mathrm{cldeg}$ grating to that of a 'standard' 2 c/deg grating, at contrasts of 10, 20, 30 and $40 \%$. Perfect matches would fall on the dotted line with a slope of one. Squares represent matches made just before and circles matches made two to three days after a depot injection of neuroleptic. Data points are means of 10 settings by each of eight subjects. Pre-injection mean standard errors ranged from 1.21 (at $10 \%$ contrast) to 2.77 (at 40\%); post-injection from 2.36 (at $20 \%$ contrast) to 2.57 (at $10 \%$ ). with a slope of one. The data are very close to this, suggesting that, in this condition, subjects could perform the task as instructed. Since the drug would be expected to affect the perception of both gratings equally, the preand post-injection matches should be similar, as indeed they are, suggesting that the subjects were using similar criteria in both test sessions. The Subjects (8) x Drug Status (2) x Contrast (4) ANOVA showed no significant effects of drug status $(\mathrm{df}=1,7 ; \mathrm{F}<1 ; \mathrm{p}>0.05)$, and no significant interaction between drug status and contrast $(\mathrm{df}=3,21 ; \mathrm{F}=1.16$; $\mathrm{p}>0.05)$, though there was a predictable highly significant effect of varying contrast $(\mathrm{df}=3,21 ; \mathrm{F}=426.2 ; \mathrm{p}<0.001)$.

Figure 2 shows the matches to the contrast of the medium $(2 \mathrm{c} / \mathrm{deg})$ spatial frequency grating of the low $(0.5 \mathrm{c} / \mathrm{deg})$ and high (8 c/deg) gratings. Different contrasts were needed to match the high and the low frequency gratings to the medium, regardless of drug status. However, the important feature

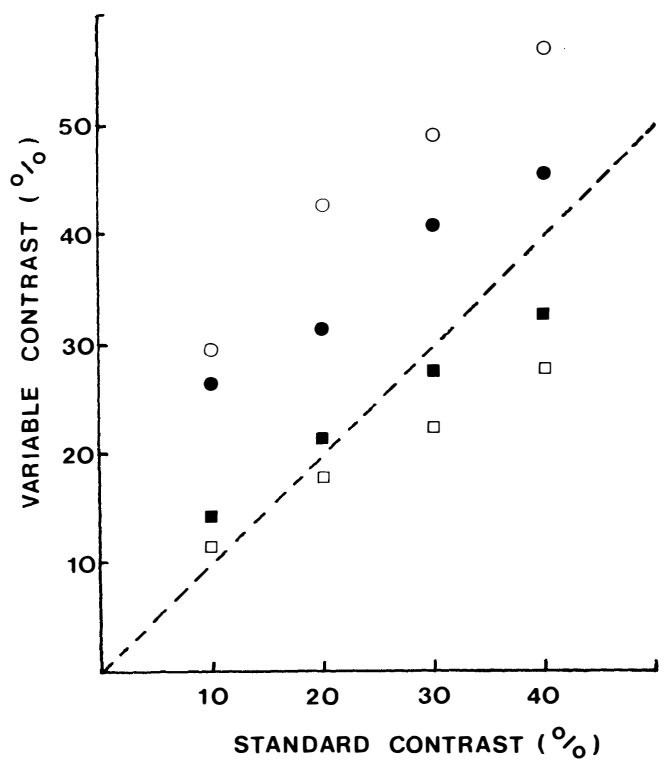

Fig. 2. The contrasts required to match the apparent contrast of a 'variable' grating (either $0.5 \mathrm{or} 8 \mathrm{c} / \mathrm{deg}$ ) to that of a 'standard' 2 cldeg grating. Open symbols are pre-, filled symbols post-injection matches. Circles 0.5, squares $8 \mathrm{c} / \mathrm{deg}$. Pre-injection mean standard errors ranged from 2.31 (at 10\% contrast, for $8 \mathrm{cldeg}$ ) to 8.43 (at $40 \%$, for $0.5 \mathrm{cldeg}$ ); post injection from 1.94 (at $10 \%, 8 \mathrm{c} / \mathrm{deg}$ ) to $6.78($ at $30 \%, 0.5 \mathrm{c} / \mathrm{deg})$. Other details as for Figure 1. 


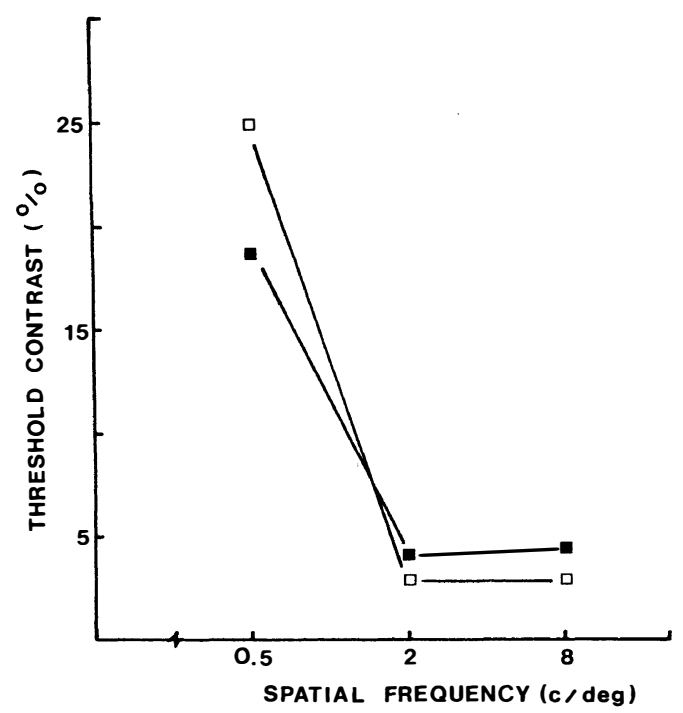

Fig. 3. Threshold contrasts for three spatial frequencies, 0.5, 2 and $8 \mathrm{c} / \mathrm{deg}$, before and after a depot injection of neuroleptic. Open symbols pre-, filled symbols post-injection. Pre-injection mean standard errors ranged from 0.66 ( $8 \mathrm{cldeg})$ to $2.76(2 \mathrm{c} / \mathrm{deg})$; postinjection 0.85 (8 c/deg) to 2.72 (2 c/deg). Other details as for Figure 1.

of the data is that the injection affects the matches for the two spatial frequencies in opposite ways. Thus more contrast was required to match that of the high spatial frequency to the medium after injection, and less to match that of the low spatial frequency to the medium, than before injection. The effect of the injection rises with the contrast of the standard. From the Subjects (8) x Contrast (4) $\mathrm{x}$ Drug Status (2) x Spatial Frequency ANOVA, the main effects of contrast $(\mathrm{df}=$ $3,21 ; \mathrm{F}=39.9 ; \mathrm{p}<0.001)$ and spatial frequency $(\mathrm{df}=1,7 ; \mathrm{F}=10.2 ; \mathrm{p}<0.05)$ were significant. The only interaction to reach significance was that of the drug status $\mathrm{x}$ spatial frequency $(\mathrm{df}=1,7 ; \mathrm{F}=14.92 ; \mathrm{p}<0.01)$, reflecting the opposite effects of the injection on the matches for the two spatial frequencies.

\section{Contrast thresholds}

Figure 3 shows contrast thresholds for the three spatial frequencies before and after injection. After injection subjects were more sensitive to the low and less sensitive to the medium and high spatial frequencies. Confirming this impression, the Subjects (8) $\mathrm{x}$ Drug Status (2) x Spatial Frequency (3)
ANOVA, showed (as well as a significant effect of spatial frequency $(\mathrm{df}=2,14 ; \mathrm{F}=55.9$; $\mathrm{p}<0.01)$ a significant interaction between drug status and spatial frequency $(\mathrm{df}=2,14$; $\mathrm{F}=10.44 ; \mathrm{p}<0.01)$.

\section{Pupillometry}

The mean pupil diameters measured at two luminances before and after injection are shown in Figure 4. The diameters measured in the two sessions are very similar, so that druginduced changes in the pupil (and so in retinal illuminance) cannot account for the changes in the perception of contrast found in the study.

\section{Discussion}

The contrast threshold and contrast matching data give a coherent picture. The effect of the depot injection of neuroleptic was to increase sensitivity to the lower spatial frequency and to reduce the amount of contrast needed to match it to the medium spatial frequency, while it had the opposite effect on the higher spatial frequency. The drug also reduced sensitivity to the medium spatial frequency,

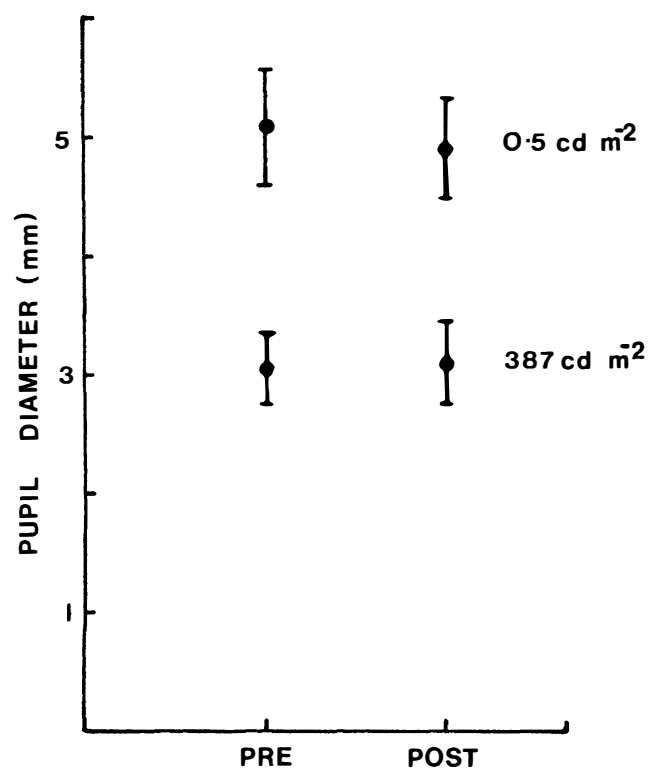

Fig. 4. Pupillary diameters at two luminances before and after a depot injection of neuroleptic. Measurements were made on averaged pupillograms, from a total of between 12 and 19 pupillograms per subject. Data points are mean diameters for eight subjects. Vertical bars are standard deviations. 
though to a smaller extent than for the higher spatial frequency, which is consistent with the change in matched contrast between these two spatial frequencies.

The contrast thresholds measured in this study are high, and suggest that subjects were using a conservative criterion. However, the changes superimposed on these thresholds by the drug are consistent. The changes in threshold produced by the presumed dopaminergic blockage are similar to those reported by Bodis-Wollner ${ }^{11}$ in his comparison of Parkinsonian patients and normal control subjects, namely a loss in sensitivity for medium and high spatial frequencies and an enhancement at low spatial frequencies. The present data suggest that such changes operate above, as well as at, detection threshold. They are consistent with the view that dopamine is important in the organisation of receptive field properties, perhaps in the retina, in human vision. The exact nature of the role of dopamine cannot be specified from the present study. One possibility is that dopamine blockage simply increases the size of the excitatory centres of receptive fields, another that there is an additional effect on contrast gain. Both effects have bren suggested as physiological bases for dark adaptation, ${ }^{23,10}$ and so the data are consistent with the luminance adaptation hypothesis of dopaminergic retinal function.

If pharmacological agents are to be tried as adjuncts to conventional environmental therapy for amblyopia, then dopamine agonists seem worth considering. Some of the studies described above suggest that they will have the opposite effects to the depot neuroleptic used here. The exact nature of these effects is not yet clear, but they appear to include an improvement in acuity or contrast sensitivity of neurones with small receptive fields. As this paper goes to press, a report has appeared of improvement, after administration of L-dopa, in human contrast sensitivity in amblyopia, which supports some of the speculations made here. ${ }^{24}$

We thank the MRC (Grant No. G1812717N) for support; Sue Beaumont for analysis of the pupil records; Ian Low for technical help; and Dr Don Early and the staff of the Industrial Therapy Organisation, Bristol, for help with recruiting subjects. Permission for the study was given by the Bristol and Weston Health District Ethical Committee.

\section{References}

${ }^{1}$ Frederick JM, Rayborn ME, Laties AM, Lam DMK, Hollyfield JG: Dopamine neurons in human retina. J Comp Neurol 1982, 210: 65-79.

${ }^{2}$ Tork I and Turner S: Histochemical evidence for a catecholoaminergic (presumably dopaminergic) projection from the ventral tegmentum to visual cortex in the cat. Neuroscience Lett 1981, 24: 215-19.

${ }^{3}$ Iuvone PM, Galli CL, Garrison-Gund CK, Neff $\mathrm{NH}$ : Light stimulates tyrosine hydroxylase activity and dopamine synthesis in retinal amacrine neurones. Science 1978, 202: 901.

${ }^{4}$ Iuvone PM, Galli CL, Neff NH: Retinal tyrosine hydroxylase: a comparison of short term and long term stimulation by light. Molecular Pharmacol 1978, 14: 1212.

${ }^{5}$ Godley BF, Flaherty AW, Wurtman RJ: The effect of light on retinal dopamine in the rat. Ann NY Acad Sci 1985, 453: 383-4.

${ }^{6}$ Thier $\mathrm{P}$ and Alder V: Action of iontophoretically applied dopamine on cat retinal ganglion cells. Brain Res 1984, 292: 109-21.

${ }^{7}$ Jensen RJ and Daw NW: Effects of dopamine and its agonists and antagonists on the receptive field properties of ganglion cells in the rabbit retina. Neuroscience 1986, 17: 837-55.

${ }^{8}$ Ehinger B: Functional role of dopamine in the retina. In: Progress in Retinal Research, Vol 2, edited by NN Osborne and GJ Chader. Pergamon Press, Oxford 1983.

${ }^{9}$ Van Nes FL and Bouman MA: Variations of contrast sensitivity with luminance. J Opt Soc Am 1967, 57: 401-40.

${ }^{10}$ Shapley RM and Enroth-Cugell C: Visual adaptation and retinal gain controls. Progress in Retinal Research, Vol 3, edited by NN Osborne and GJ Chader, 1984, 263-343.

${ }^{11}$ Bodis-Wollner I: Altered spatio-temporal contrast vision in Parkinson's disease and MPTP-treated monkeys: the role of dopamine. In: Dopaminergic mechanisms in vision. Eds: I Bodis-Wollner and M Piccolino. New York: AR Liss, Inc., 1988, pp 205-20.

${ }^{12}$ Bodis-Wollner I, Yahr MD, Thornton JA: Visual evoked potentials and the severity of Parkinson's disease. In: Research Progress in Parkinson's Disease. Eds: FC Rose and R Capildeo, Tunbridge Wells: Pitman Medical, 1981.

${ }^{13}$ Ellis CJK and Ikeda H: Evidence for retinal dopamine deficiency in Parkinson's disease. In: Dopaminergic mechanisms in vision. Eds: I Bodis-Wollner and $M$ Piccolino. New York: AR Liss, Inc., 1988, pp 239-51.

${ }^{14}$ Domenici L, Trimarchi C, Piccolino M, Fiorentini A, Maffei L: Dopaminergic drugs improve visual contrast sensitivity. Hum Neurobiol 1985, 4: 195-7.

${ }^{15}$ Harris JP and Calvert JE: Contrast, spatial frequency and test duration effects on the tilt aftereffect: implications for underlying mechanisms. Vis Res 1989, 29: 129-35. 
${ }^{16}$ Calvert JE, Harris JP, Phillipson OT: Effects of L-dopa on the tilt aftereffect with differing stimulus contrast and test duration. Clin Vis Sci 1990, 5: 87-90.

${ }^{17}$ Calvert JE, Harris JP, Phillipson OT: Probing the visual system of Parkinson's disease and chronic schizophrenic patients on depot neuroleptic using the tilt aftereffect. (In preparation.)

${ }^{18}$ Harris JP, Low I, Makepeace APW: A programmable waveform generator to produce stationary and moving gratings for visual research. J Physiol 1982, 327: $15 \mathrm{P}$.

${ }^{19}$ Mariani AP, Kolb H, Nelson R: Dopamine-containing cells of the rhesus monkey retina parallel rods in spatial distribution. Brain Res 1984, 322: 1-7.

${ }^{20}$ Nguyen-Legros J and Savy C: Dopamine innervation of the vertebrate retina: morphological studies. In: Dopaminergic mechanisms in vision. Eds: I Bodis-Wollner and M Piccolino. New York: AR Liss Inc., 1988, pp 1-17.

${ }^{21}$ Beaumont SM, Harris JP, Leendertz J A, Phillipson OT: The pupillary light reflex in mild Parkinson's disease. Clin Vis Sci 1987, 2: 123-9.

${ }^{22}$ Jorgensen A, Hansen V, Larsen VD, Khan AR: Metabolism, distribution and excretion of flupenthixol. Acta Pharmacol Toxicol 1969, 27: 301-13.

${ }^{23}$ Barlow HB, Fitzhugh R, Kuffler SW: Change of organisation in the receptive fields of the cat's retina during dark adaptation. J Physiol 1957, 137: $338-154$

${ }^{24}$ Gottlob I and Stangler-Zuschrott E: Effect of levodopa on contrast sensitivity and scotomas in human amblyopia. Invest Ophthalmol Vis Sci 1990, 31: 776-80. 